\title{
Purpura Fulminans in an Asplenic Intravenous Drug User
}

\author{
Emily S. Nyers, MD; Rachael H. Kappius, MD; Laura S. Winterfield, MD; Dirk M. Elston, MD
}

\section{PRACTICE POINTS}

- Capnocytophaga species are fastidious, slowgrowing microorganisms. It is important, therefore, to maintain a high degree of suspicion and alert the microbiology laboratory to increase the likelihood of isolation.

- Patients should be cautioned regarding the need for prophylactic antibiotics in the event of an animal bite; asplenic patients are at particular risk for infection.

- In patients with severe purpura fulminans and a gangrenous limb, it is important to allow adequate time for demarcation of gangrene and not rush to amputation.

To the Editor:

A 56-year-old man with a history of opioid abuse and splenectomy decades prior due to a motor vehicle accident was brought to an outside emergency department with confusion, slurred speech, and difficulty breathing. Over the next few days, he became febrile and hypotensive, requiring vasopressors. Clinical laboratory testing revealed a urine drug screen positive for opioids and a low platelet count in the setting of a rapidly evolving retiform purpuric rash.

The patient was transferred to our institution 6 days after initial presentation with primary diagnoses of septic shock with multiorgan failure and disseminated intravascular coagulation (DIC). Blood cultures were positive for gram-negative rods. After several days of broad-spectrum antibiotics and supportive care, cultures were reported as positive for Capnocytophaga canimorsus. Upon further questioning, the patient's wife reported that the couple had a new puppy and that the patient often allowed the dog to bite him playfully and lick abrasions on his hands and legs. He had not received medical treatment for any of the dog's bites.

On initial examination at the time of transfer, the patient's skin was remarkable for diffuse areas of stellate and retiform purpura with dusky centers and necrosis of the nasal tip and earlobes. Both hands were purpuric, with necrosis of the fingertips (Figure 1A). The flank was marked by large areas of full-thickness sloughing of the skin (Figure 1B). The lower extremities were edematous, with some areas of stellate purpura and numerous large bullae that drained straw-colored fluid (Figure 1C). Lower extremity pulses were found with Doppler ultrasonography.

Given the presence of rapidly developing retiform purpura in the clinical context of severe sepsis, purpura fulminans (PF) was the primary consideration in the differential diagnosis. Levamisole-induced necrosis syndrome also was considered because of necrosis of the ears and nose as well as the history of substance use; however, the patient was not known to have a history of cocaine abuse, and a test of antineutrophil cytoplasmic antibody was negative.

A punch biopsy of the abdomen revealed intravascular thrombi with epidermal and sweat gland necrosis, consistent with PF (Figure 2). Gram, Giemsa, and Gomori methenamine-silver stains were negative for organisms. Tissue culture remained negative. Repeat blood cultures demonstrated Candida parapsilosis fungemia. Respiratory culture was positive for budding yeast.

The patient was treated with antimicrobials, intravenous argatroban, and subcutaneous heparin. Purpura and bullae on the trunk slowly resolved with systemic therapy

From the Department of Dermatology and Dermatologic Surgery, Medical University of South Carolina, Charleston.

The authors report no conflict of interest.

Correspondence: Emily S. Nyers, MD, 135 Rutledge Ave, MSC 578, Charleston, SC 29425 (nyers@musc.edu).

doi: $10.12788 /$ cutis.0396 

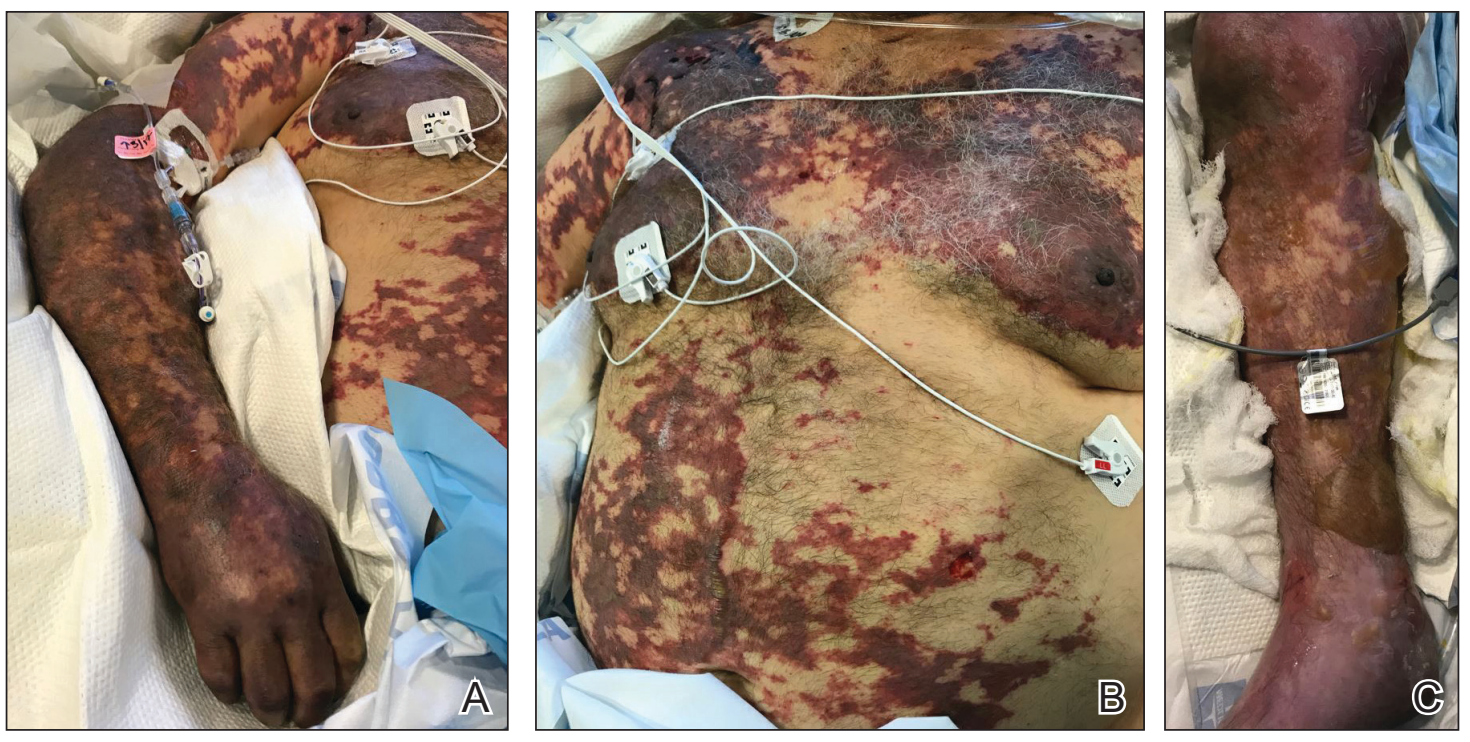

FIGURE 1. A, Retiform purpura with erosions and dusky appearance of the hand and digits. B, Extensive retiform purpura and early necrosis across the chest and abdomen. C, Large bullae were present on the lower leg.

and wound care with petrolatum and nonadherent dressings. However, lesions on the nasal tip, all fingers of both hands, and several toes evolved into dry gangrene. The hospital course was complicated by renal failure requiring continuous renal replacement therapy; respiratory failure requiring ventilator support; and elevated levels of liver enzymes, consistent with involvement of the hepatic microvasculature.

The patient was in the medical intensive care unit at our institution for 2 weeks and was transferred to a burn center for specialized wound care. At transfer, he was still on a ventilator and receiving continuous renal replacement therapy. Subsequently, the patient required a left above-the-knee amputation, right below-the-knee amputation, and amputation of several digits of the upper extremities. In the months after the amputations, he required multiple stump revisions and experienced surgical site infections that complicated healing.

Purpura fulminans is an uncommon syndrome characterized by intravascular thrombosis and hemorrhagic infarction of the skin. The condition commonly is associated with septic shock, causing vascular collapse and DIC. It often develops rapidly.

Because of associated high mortality, it is important to differentiate $\mathrm{PF}$ from other causes of cutaneous retiform purpura, including other causes of thrombosis and large vessel vasculitis. Leading causes of PF include infection and hereditary or acquired deficiency of protein $\mathrm{C}$, protein $\mathrm{S}$, or antithrombin III. Regardless of cause, biopsy results demonstrate vascular thrombosis out of proportion to vasculitis. The mortality rate is $42 \%$ to $50 \%$. The incidence of postinfectious sepsis sequelae in PF is higher than in survivors of sepsis only, especially amputation. ${ }^{1-3}$ Most patients do not die from complications of sepsis

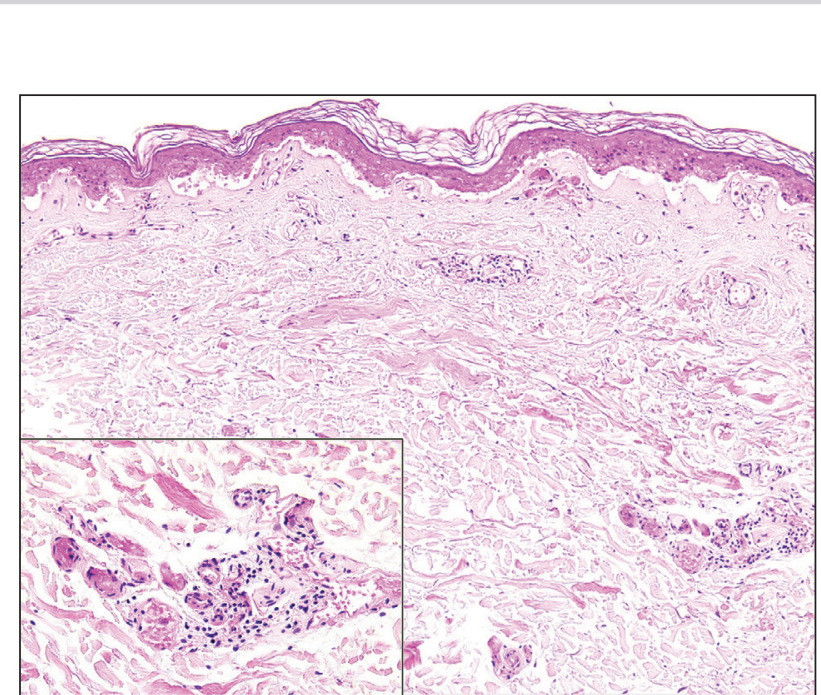

FIGURE 2. A punch biopsy of the abdomen revealed intravascular thrombi, epidermal detachment, and epidermal and sweat gland necrosis, consistent with purpura fulminans (H\&E, original magnification $\times 100$ [inset, original magnification $\times 200]$ ).

but from sequelae of the hypercoagulable and prothrombotic state associated with $\mathrm{PF}^{4}$ Hemorrhagic infarction can affect the kidneys, brain, lungs, heart, eyes, and adrenal glands (ie, necrosis, namely WaterhouseFriderichsen syndrome). ${ }^{5}$

The most common infectious cause of PF is sepsis secondary to Neisseria meningitidis, with as many as $25 \%$ of infected patients developing PF. ${ }^{6}$ Streptococcus pneumoniae is another common cause. Other important causative organisms include Streptococcus pyogenes; Staphylococcus aureus (in the setting of intravenous substance use); Klebsiella oxytoca; Klebsiella aerogenes; rickettsial organisms; and viruses, including cytomegalovirus 
and varicella-zoster virus. ${ }^{2,7-13}$ Two earlier cases associated with Capnocytophaga were characterized by concomitant renal failure, metabolic acidosis, hemolytic anemia, and DIC. ${ }^{14}$

It is estimated that Capnocytophaga causes $11 \%$ to $46 \%$ of all cases of sepsis ${ }^{15}$; sepsis resulting from Capnocytophaga has extremely poor outcomes, with mortality reaching as high as $60 \%$. The organism is part of the normal oral flora of cats and dogs, and a bite (less often, a scratch) is the cause of most Capnocytophaga infections. The clinical spectrum of $C$ canimorsus infection associated with dog saliva exposure more commonly includes cellulitis at or around the site of inoculation, meningitis, and endocarditis. ${ }^{16}$

Although patients affected by PF can be young and healthy, several risk factors for PF have been identified ${ }^{2,6,16}$ : asplenia, an immunocompromised state, systemic corticosteroid use, cirrhosis, and alcoholism. Asplenic patients have been shown to be particularly susceptible to systemic Capnocytophaga infection; when bitten by a dog, they should be treated with prophylactic antibiotics to cover Capnocytophaga. ${ }^{17}$ Immunocompetent patients rarely develop severe infection with Capnocytophaga. ${ }^{16,18,19}$ The complement system in particular is critically important in defending against $C$ canimorsus. ${ }^{20}$

The underlying pathophysiology of acute infectious $\mathrm{PF}$ is multifactorial, encompassing increased expression of procoagulant tissue factor by monocytes and endothelial cells in the presence of bacterial pathogens. Dysfunction of protein $C$, an anticoagulant component of the coagulation cascade, often is cited as a crucial derangement leading to the development of a prothrombotic state in acute infectious PF. ${ }^{21}$ Serum protein $\mathrm{S}$ and antithrombin deficiency also can play a role. ${ }^{22}$ Specific in vitro examination of $C$ canimorsus has revealed a protease that catalyzes $\mathrm{N}$-terminal cleavage of procoagulant factor $X$, resulting in loss of function. ${ }^{15}$

Retiform purpura is a hallmark feature of $\mathrm{PF}$, often beginning as nonblanching erythema with localized edema and petechiae before evolving into the characteristic stellate lesions with hemorrhagic bullae and subsequent necrosis. ${ }^{23}$ Pathologic examination reveals microthrombi involving arterioles and smaller vessels. ${ }^{24}$ There typically is laboratory evidence of DIC in PF, including elevated prothrombin time and partial thromboplastin time, thrombocytopenia, elevated D-dimer, and a decreased fibrinogen level. ${ }^{6,23}$

Capnocytophaga bacteria are challenging to grow on standard culture media. Optimal media for growth include $5 \%$ sheep's blood and chocolate agar. ${ }^{16}$ Polymerase chain reaction can identify Capnocytophaga; in cases in which blood culture does not produce growth, 16S ribosomal RNA gene sequencing of tissue from skin biopsy has identified the pathogen. ${ }^{25}$

Some Capnocytophaga isolates have been shown to produce beta-lactamase; individual strains can be resistant to penicillins, cephalosporins, and imipenem. ${ }^{26}$
Factors associated with an increased risk for death include decreased leukocyte and platelet counts and an increased level of arterial lactate. ${ }^{27}$

Empiric antibiotic therapy for Capnocytophaga sepsis should include a beta-lactam and beta-lactamase inhibitor, such as piperacillin-tazobactam. Management of DIC can include therapeutic heparin or low-molecular-weight heparin and prophylactic platelet transfusion to maintain a pre-established value. ${ }^{28-30}$ Debridement should be conservative; it is important to wait for definite delineation between viable and necrotic tissue, ${ }^{31}$ which might take several months. ${ }^{32}$ Human skin allografts, in addition to artificial skin, are utilized as supplemental therapy for more rapid wound closure after removal of necrotic tissue. ${ }^{33,34}$ Hyperoxygenated fatty acids have been noted to aid in more rapid wound healing in infants with $\mathrm{PF}^{35}$

Fresh frozen plasma is one method to replace missing factors, but it contains little protein C. ${ }^{36}$ Outcomes with recombinant human activated protein $\mathrm{C}$ (drotrecogin alfa) are mixed, and studies have shown no benefit in reducing the risk for death. ${ }^{37,38}$ Protein $\mathrm{C}$ concentrate has shown therapeutic benefit in some case reports and small retrospective studies. ${ }^{4}$ In one case report, protein $\mathrm{C}$ concentrate and heparin were utilized in combination with antithrombin III. ${ }^{21}$

Hyperbaric $\mathrm{O}_{2}$ might be of benefit when initiated within 5 days after onset of PF. However, hyperbaric $\mathrm{O}_{2}$ does carry risk; $\mathrm{O}_{2}$ toxicity, barotrauma, and barriers to timely resuscitation when the patient is inside the pressurized chamber can occur. ${ }^{2}$

There is a single report of successful use of the vasodilator iloprost for meningococcal PF without need for surgical intervention; the team also utilized topical nitroglycerin patches on the fingers to avoid digital amputation. ${ }^{39}$ Epoprostenol, tissue plasminogen activator, and antithrombin have been utilized in cases of extensive PF. Fibrinolytic therapy might have some utility, but only in a setting of malignancy-associated DIC. ${ }^{40}$

Treatment of acute infectious PF lacks a high level of evidence. Options include replacement of anticoagulant factors, anticoagulant therapy, hyperbaric $\mathrm{O}_{2}$, topical and systemic vasodilators, and, in the setting of underlying cancer, fibrinolytics. Even with therapy, prognosis is guarded.

\section{REFERENCES}

1. Ghosh SK, Bandyopadhyay D, Dutta A. Purpura fulminans: a cutaneous marker of disseminated intravascular coagulation. West J Emerg Med. 2009; 10:41.

2. Ursin Rein P, Jacobsen D, Ormaasen V, et al. Pneumococcal sepsis requiring mechanical ventilation: cohort study in 38 patients with rapid progression to septic shock. Acta Anaesthesiol Scand. 2018;62:1428-1435. doi:10.1111/aas

3. Contou D, Canoui-Poitrine F, Coudroy R, et al; Hopeful Study Group. Long-term quality of life in adult patients surviving purpura fulminans: an exposed-unexposed multicenter cohort study. Clin Infect Dis. 2019;69:332-340. doi:10.1093/cid/ciy901

4. Chalmers E, Cooper P, Forman K, et al. Purpura fulminans: recognition, diagnosis and management. Arch Dis Child. 2011;96:1066-1071. doi:10.1136/adc.2010.199919 
5. Karimi K, Odhav A, Kollipara R, et al. Acute cutaneous necrosis: a guide to early diagnosis and treatment. J Cutan Med Surg. 2017;21:425-437. doi:10.1177/1203475417708164

6. Colling ME, Bendapudi PK. Purpura fulminans: mechanism and management of dysregulated hemostasis. Transfus Med Rev. 2018;32:69-76. doi:10.1016/j.tmrv.2017.10.001

7. Kankeu Fonkoua L, Zhang S, Canty E, et al. Purpura fulminans from reduced protein $\mathrm{S}$ following cytomegalovirus and varicella infection. Am J Hematol. 2019;94:491-495. doi:10.1002/ajh.25386

8. Okuzono S, Ishimura M, Kanno S, et al. Streptococcus pyogenespurpura fulminans as an invasive form of group A streptococcal infection. Ann Clin Microbiol Antimicrob. 2018;17:31. doi:10.1186 /s12941-018-0282-9

9. Gupta D, Chandrashekar L, Srinivas BH, et al. Acute infectious purpura fulminans caused by group A $\beta$-hemolytic Streptococcus: an uncommon organism. Indian Dermatol Online J. 2016;7:132-133. doi:10.4103 /2229-5178.178093

10. Saini S, Duncan RA. Sloughing skin in intravenous drug user. IDCases. 2018;12:74-75. doi:10.1016/j.idcr.2018.03.007

11. Tsubouchi N, Tsurukiri J, Numata J, et al. Acute infectious purpura fulminans caused by Klebsiella oxytoca. Intern Med. 2019;58:1801-1802. doi:10.2169/internalmedicine.2350-18

12. Yamamoto S, Ito R. Acute infectious purpura fulminans with Enterobacter aerogenes post-neurosurgery. IDCases. 2019;15:e00514. doi:10.1016/j.idcr.2019.e00514

13. Dalugama C, Gawarammana IB. Rare presentation of rickettsial infection as purpura fulminans: a case report. J Med Case Rep. 2018;12:145. doi:10.1186/s13256-018-1672-5

14. Kazandjieva J, Antonov D, Kamarashev J, et al. Acrally distributed dermatoses: vascular dermatoses (purpura and vasculitis). Clin Dermatol. 2017;35:68-80. doi:10.1016/j.clindermatol.2016.09.013

15. Hack K, Renzi F, Hess E, et al. Inactivation of human coagulation factor $\mathrm{X}$ by a protease of the pathogen Capnocytophaga canimorsus. J Thromb Haemost. 2017;15:487-499. doi:10.1111/jth.13605

16. Zajkowska J, Król M, Falkowski D, et al. Capnocytophaga canimorsusan underestimated danger after dog or cat bite - review of literature. Przegl Epidemiol. 2016;70:289-295.

17. Di Sabatino A, Carsetti R, Corazza GR. Post-splenectomy and hyposplenic states. Lancet. 2011;378:86-97. doi:10.1016 /S0140-6736(10)61493-6

18. Behrend Christiansen C, Berg RMG, Plovsing RR, et al. Two cases of infectious purpura fulminans and septic shock caused by Capnocytophaga canimorsus transmitted from dogs. Scand J Infect Dis. 2012;44:635-639. doi:10.3109/00365548.2012.672765

19. Ruddock TL, Rindler JM, Bergfeld WF. Capnocytophaga canimorsus septicemia in an asplenic patient. Cutis. 1997;60:95-97.

20. Mantovani E, Busani S, Biagioni E, et al. Purpura fulminans and septic shock due to Capnocytophaga canimorsus after dog bite: a case report and review of the literature. Case Rep Crit Care. 2018;2018:7090268. doi:10.1155/2018/7090268

21. Bendapudi PK, Robbins A, LeBoeuf N, et al. Persistence of endothelial thrombomodulin in a patient with infectious purpura fulminans treated with protein C concentrate. Blood Adv. 2018; 2:2917-2921. doi:10.1182/bloodadvances.2018024430

22. Lerolle N, Carlotti A, Melican K, et al. Assessment of the interplay between blood and skin vascular abnormalities in adult purpura fulminans. Am J Respir Crit Care Med. 2013;188:684-692. doi:10.1164/rccm.201302-0228OC.

23. Thornsberry LA, LoSicco KI, English JC III. The skin and hypercoagulable states. J Am Acad Dermatol. 2013;69:450-462. doi:10.1016/j.jaad.2013.01.043
24. Adcock DM, Hicks MJ. Dermatopathology of skin necrosis associated with purpura fulminans. Semin Thromb Hemost. 1990;16:283-292. doi:10.1055/s-2007-1002681

25. Dautzenberg KHW, Polderman FN, van Suylen RJ, et al. Purpura fulminans mimicking toxic epidermal necrolysis-additional value of 16S rRNA sequencing and skin biopsy. Neth J Med. 2017;75:165-168.

26. Zangenah S, Andersson AF, Özenci V, et al. Genomic analysis reveals the presence of a class D beta-lactamase with broad substrate specificity in animal bite associated Capnocytophaga species. Eur J Clin Microbiol Infect Dis. 2017;36:657-662. doi:10.1007/s10096-016-2842-2

27. Contou D, Sonneville R, Canoui-Poitrine F, et al; Hopeful Study Group. Clinical spectrum and short-term outcome of adult patients with purpura fulminans: a French multicenter retrospective cohort study. Intensive Care Med. 2018;44:1502-1511. doi:10.1007/s00134-018-5341-3

28. Zenz W, Zoehrer B, Levin $\mathrm{M}$, et al; International Paediatric Meningococcal Thrombolysis Study Group. Use of recombinant tissue plasminogen activator in children with meningococcal purpura fulminans: a retrospective study. Crit Care Med. 2004;32:1777-1780. doi:10.1097/01.ccm.0000133667.86429.5d

29. Wallace JS, Hall JC. Use of drug therapy to manage acute cutaneous necrosis of the skin. J Drugs Dermatol. 2010;9:341-349.

30. Squizzato A, Hunt BJ, Kinasewitz GT, et al. Supportive management strategies for disseminated intravascular coagulation. an international consensus. Thromb Haemost. 2016;115:896-904. doi:10.1160/TH15-09-0740

31. Herrera R, Hobar PC, Ginsburg CM. Surgical intervention for the complications of meningococcal-induced purpura fulminans. Pediatr Infect Dis J. 1994;13:734-737. doi:10.1097/00006454-199408000-00011

32. Pino PA, Román JA, Fernández F. Delayed surgical debridement and use of semiocclusive dressings for salvage of fingers after purpura fulminans. Hand (NY). 2016;11:NP34-NP37. doi:10.1177/1558944716661996

33. Gaucher S, Stéphanazzi J, Jarraya M. Human skin allografts as a useful adjunct in the treatment of purpura fulminans. J Wound Care. 2010;19:355-358. doi:10.12968/jowc.2010.19.8.77714

34. Mazzone L, Schiestl C. Management of septic skin necroses. Eur J Pediatr Surg. 2013;23:349-358. doi:10.1055/s-0033-1352530

35. Pérez-Acevedo G, Torra-Bou JE, Manzano-Canillas ML, et al. Management of purpura fulminans skin lesions in a premature neonate with sepsis: a case study. J Wound Care. 2019;28:198-203. doi:10.12968 /jowc.2019.28.4.198

36. Kizilocak H, Ozdemir N, Dikme G, et al. Homozygous protein C deficiency presenting as neonatal purpura fulminans: management with fresh frozen plasma, low molecular weight heparin and protein C concentrate. J Thromb Thrombolysis. 2018;45:315-318. doi:10.1007/s11239-017-1606-x

37. Ranieri VM, Thompson BT, Barie PS, et al; PROWESS-SHOCK Study Group. Drotrecogin alfa (activated) in adults with septic shock. N Engl J Med. 2012;366:2055-2064. doi:10.1056/NEJMoa1202290

38. Bernard GR, Vincent J-L, Laterre P-F, et al; Recombinant Human Protein C Worldwide Evaluation in Severe Sepsis (PROWESS) Study Group. Efficacy and safety of recombinant human activated protein C for severe sepsis. N Engl J Med. 2001;344:699-709. doi:10.1056 /NEJM200103083441001

39. Hage-Sleiman M, Derre N, Verdet C, et al. Meningococcal purpura fulminans and severe myocarditis with clinical meningitis but no meningeal inflammation: a case report. BMC Infect Dis. 2019;19:252. doi:10.1186/s12879-019-3866-x

40. Levi M, Toh $\mathrm{CH}$, Thachil J, et al. Guidelines for the diagnosis and management of disseminated intravascular coagulation. British Committee for Standards in Haematology. Br J Haematol. 2009; 145:24-33. doi:10.1111/j.1365-2141.2009.07600.x 